\section{Improving AEFI Surveillance in India}

We read with interest the position paper of Indian Academy of Pediatrics on AEFI surveillance [1]. In addition to the web -based facilities and SMS alerts, we propose that a mobile application should be developed for prompt reporting of AEFI. Mobile app is immensely useful in expedited delivery of such information to the concerned authorities by the caregiver and can function offline. It can be linked directly to the database which could reduce the missing cases. There are 898 million mobile subscribers in India with more than 50 per cent accessing the internet only via mobile devices [2]. Apart from this, a toll-free number can be created for easier reporting of adverse events. The operator can do realtime data entry, thus improving reporting.
VidUShi MahaJan AND *Shiv SAJan SAINI Department of Pediatrics, Government Medical College and Hospital, and Neonatology Unit, Department of Pediatrics, Post Graduate Institute of Medical and Research, Chandigarh, India. sajansaini1@gmail.com

\section{REFERENCES}

1. Chitkara AJ, Thacker N, Vashsihtha VM, Bansal CP, Gupta SG. Advisory Committee on Vaccines and Immunization Practices, Indian Academy of Pediatrics. Adverse Event Following Immunization (AEFI) surveillance in India: Position paper of Indian Academy of Pediatrics, 2013. Indian Pediatr. 2013;50:739-41.

2. Vaidyanathan R. Is 2012 the Year for India's Internet? Mumbai: BBC News; 2012 [cited 201320 June]; Available from: http://www.bbc.co.uk/news/business-16354076. Accessed December 1, 2013.

\section{Donkey's Milk Feeding in Newborn: Myths and Facts}

Donkey's milk is being given to newborns in certain communities of Tamilnadu and Puducherry [1]. When a baby is born, the grandmother in the family administers donkey's milk to the newborn, the myth being that it enhances growth, promotes brain development, and improves voice. It is also perceived to be an effective remedy for cough, liver problems, loss of appetite and exhaustion [2]. Neonatologists and other healthcare personnel worldwide insist on exclusive breastfeeding for the first six months of life and simultaneously warn against harmful and unhygienic feeding practices.

We witnessed two children in our hospital who expired due to donkey's milk aspiration. The first was a term male baby delivered by spontaneous vaginal delivery with a birth weight of $2.6 \mathrm{~kg}$ and received mother's milk within half an hour of delivery, and was accepting feeds adequately. On 2nd day of life the neonate was brought with cyanosis, bradycardia and apnea. Copious amount of milk was found in the oral activity and the neonate expired subsequently despite our resuscitative efforts. Another term male baby with birth weight of $2.9 \mathrm{~kg}$, who was accepting sufficient breast milk feeds, was brought to us with no signs of life; examination revealed aspiration of milk. In both the infants, the parents accepted that they had tried to feed them donkey's milk.

The promotion of breast milk is emphasized worldwide, as it is species specific, enhances brain development, prevents infection and improves motherchild bonding [3]. There is no scientific evidence for the use of donkey's milk in newborns. Myths and superstitions should not be allowed at the cost of safe feeding practices. Cognizance of prevailing harmful feeding practices in various communities and parts of the country should be a first step. Mothers' education and community awareness in this direction should be actively promoted as a deterrent to these practices. Exclusive breast-feeding in the first six months of life is the dictum. The grandma recipe of giving donkey's milk should give way to safe feeding practices. Not only the neonatologists and health workers, but the community as a whole should play a definitive, positive and continuing role. 